\title{
Electrically-controlled Neuron-like Spiking Regimes in Vertical-cavity Surface-emitting Lasers at Ultrafast Rates
}

\author{
Joshua Robertson, Ewan Wade, and Antonio Hurtado
}

\begin{abstract}
We report experimentally on the electricallycontrolled, tunable and repeatable neuron-like spiking regimes generated in an optically-injected vertical-cavity surface-emitting laser (VCSEL) operating at the telecom wavelength of $1300 \mathrm{~nm}$. These fast spiking dynamics (obtained at sub-nanosecond speed rates) demonstrate different behaviours observed in biological neurons such as thresholding, phasic and tonic spiking and spike rate and spike latency coding. The spiking regimes are activated in response to external stimuli (with controlled strengths and temporal duration) encoded in the bias current applied to a VCSEL subject to continuous wave (CW) optical injection (OI). These results reveal the prospect for fast $(>7$ orders of magnitude faster than neurons), novel, electrically-controlled spiking photonic modules for future neuromorphic computing platforms.
\end{abstract}

Index Terms-Vertical-cavity surface-emitting lasers (VCSELs), neuromorphic photonics, photonic neurons, spiking, photonic spiking processing.

\section{INTRODUCTION}

$\mathrm{R}$ ESEARCH efforts towards novel realisations of neuronal models and computing paradigms have traditionally been achieved using electronic techniques such as CMOS chips [14]. Projects such as the Neurogrid project at Standford [1], HICANN at the University of Heidelberg [2], TrueNorth at IBM [3] and SpiNNaker at the University of Manchester [4] have all demonstrated that this technology is capable of impressive levels of interconnectivity and spike communication in neural-inspired circuits. However, roughly since the turn of the millennia [5], photonic realisations, that take advantage of the unique properties of light, have emerged towards the neuromorphic research effort. Photonic technologies, that grant potentially large bandwidths, low cross talk elements and high integration, possess impressive ultrafast speeds compared to the spiking speed of biological neurons ( $>7$ orders of magnitude faster). Over the past decade

This work was supported in part by the Office of Naval Research Global (ONRG) under Grant ONRGNICOP-N62909-18-1-2027) and the UK's EPSRC Doctoral Training Partnership (EP/N509760).

The authors are with the Institute of Photonics, SUPA Department of Physics, University of Strathclyde, Technology and Innovation Centre, 99 George Street, G1 1RD, Glasgow (United Kingdom) (joshua.robertson@strath.ac.uk; antonio.hurtado@strath.ac.uk). the toolkit of neuromorphic photonic devices has expanded to include technologies such as photonic crystal structures [6,7], resonant tunneling diode-laser diode (RTD-LD) coupled systems [8,9], fibre lasers [10,11], semiconductor optical amplifiers (SOAs) [12,13], optical modulators [14,15] and semiconductor lasers (SLs) [16-30]. SLs are one of the most prominent devices in the development of artificial optical neurons as they exhibit the diverse behaviours observed in biological neurons, such as excitability [26,31-34] and complex non-linear dynamics [35-36]. As a result, an increasing number of approaches for artificial neuronal models based on diverse types of SLs ranging from micro-disk lasers [16], micro-pillar lasers [17-19] and micro-ring lasers [20-21] to quantum dot (QD) lasers [22-24], lasers with saturable absorber sections [25] and vertical-cavity surface-emitting lasers (VCSELs) [26-29] have recently appeared (for a review see [30]).

In particular, VCSELs have attracted great research interest for use in neuromorphic photonics, given their inherent advantages, such as ultra-small footprint, low manufacturing cost, high-speed, potential for large scale integration and operation at telecommunication wavelengths, to name but a few [37,38]. The proposed use of VCSELs as artificial photonic neurons has seen the application of techniques such as polarization switching (PS) $[39,40]$ and optical injection (OI) [26-29][41-46] for the all-optical conversion of binary signals to spiking patterns. Perturbed OI has been used experimentally to demonstrate the controllable activation [41], and inhibition [42], of sub-ns excitable spike patterns in telecom-wavelength VCSELs with promising neuronal features. These controllable spiking dynamical regimes in VCSELs, created using all-optical photonic components, replicated biological neuronal responses by producing behaviours similar to thresholding (the requirement of superthreshold stimuli intensity to fire a response) and tonic spiking (the continuous firing of spiking responses across the duration of a stimulus). Furthermore, recent numerical simulations, based on the spin-flip model [47], suggest that artificial VCSEL neurons subject to dual polarized injection may have potentially improved signal quality by avoiding undesirable relaxation oscillations [45]. Dual polarized optical injection has also demonstrated numerically the successful encoding of noise-robust spike signals in two polarization separated channels simultaneously [46]. The coupling and 
interconnection of these photonic neuromorphic elements has also been explored with the successful communication of fast spiking dynamics in a cascaded transmitter-receiver VCSEL configuration $[43,44]$, and with detailed theoretical studies of multiple mutual coupling configurations $[48,49]$. The behavioural similarities that can be drawn between the investigated VCSEL-based neuronal models and biological neurons are what allow us to pursue these devices as candidates for artificial photonic neurons.

In this work we report on the novel, electrically-controlled, generation of ultrafast spiking regimes in VCSELs operating at telecommunication wavelengths $(1300 \mathrm{~nm})$. We demonstrate that short temporal perturbations (stimuli) encoded in the applied bias current of a VCSEL, subject to continuous wave $(\mathrm{CW})$ optical injection (OI), can trigger the firing of controllable spiking regimes. The results are produced by combining the driving current applied to the VCSEL and either an electrical or optical (wavelengthindependent) amplitude-varying signal using a bias tee. The system responds by firing a controllable number of $\sim 100 \mathrm{ps}$ wide spikes at sub-nanosecond inter-spiking-intervals. This electronically-controlled photonic neuronal model enables the integration of multiple photonic inputs from diverse sources at different wavelengths for interconnected, ultrafast, spiking neuromorphic systems. The features of these systems are highly desirable in the search for novel neuronal computing paradigms with prospects of contributing to new future processing architectures.

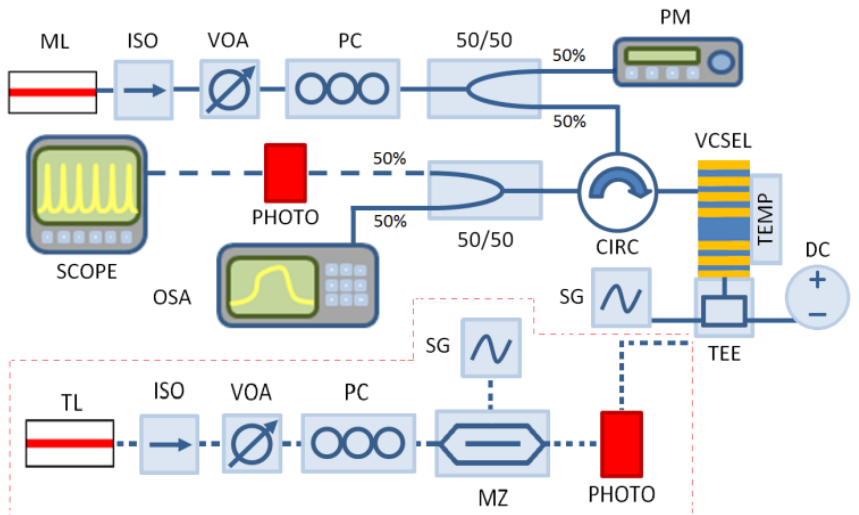

Fig. 1. Diagram of the setup used for the electrical activation of sub-nanosecond spiking regimes in a VCSEL neuronal model at $1300 \mathrm{~nm}$. The external stimuli are injected into the VCSEL's bias current using either an electrical signal generator directly connected to a bias tee or an amplitude-modulated optical signal converted to the electrical domain via a photodetector and connected to a bias tee (red dashed box). In both cases the bias tee combines the externally generated electrical stimuli with the DC bias current applied to the device. $\mathrm{ML}=$ Master Laser, $\mathrm{ISO}=$ Optical Isolator, VOA $=$ Variable Optical Attenuator, $\mathrm{PC}=$ Polarization Controller, $\mathrm{PM}=$ Power Meter, $\mathrm{SG}=$ Signal Generator, TEMP $=$ Temperature Controls, TEE $=$ Bias Tee, $\mathrm{DC}=$ Laser Diode Driver, $\mathrm{PHOTO}=$ Photodetector, $\mathrm{CIRC}=$ Circulator, $\mathrm{SCOPE}=$ Oscilloscope, $\mathrm{OSA}=$ Optical Spectrum Analyzer, $\mathrm{TL}=$ Tunable laser, $\mathrm{MZ}=$ Mach Zehnder Amplitude Modulator.

\section{EXPERIMENTAL SETUP}

The experimental setup used in this work is shown in Fig. 1. $\mathrm{CW}$ orthogonally-polarized light from an external tunable laser (Master laser, ML) with controlled intensity and fixed frequency detuning $(\Delta f)$ was injected into the VCSEL used in the experiments. The latter was a commercially available fibre-pigtailed device $\left(\right.$ RayCan $\left.^{\mathrm{TM}}\right)$ operating at the telecommunication wavelength of $1300 \mathrm{~nm}$ and with a measured threshold current $\left(\mathrm{I}_{\mathrm{th}}\right)$ of $0.70 \mathrm{~mA}$ at $293 \mathrm{~K}$. The device's spectrum (see Fig 2(a)) showed two lasing modes corresponding to the two orthogonal polarizations of the VCSEL's fundamental transverse mode. These are labelled as parallel $\left(\lambda_{y}\right)$ and orthogonal $\left(\lambda_{x}\right)$ modes in Fig 2(a). Throughout the experiments the VCSEL was electrically biased with a current of $6.0 \mathrm{~mA}$ and its temperature was kept constant and equal at $293 \mathrm{~K}$. The parallel (orthogonal) mode was the main lasing (subsidiary attenuated) mode for all applied bias currents above threshold and no current-induced polarization switching was observed. The frequency detuning is defined as the frequency difference between the resonant frequencies of the ML $\left(f_{i n j}\right)$ and that of the VCSEL's orthogonal mode $\left(f_{x}\right), \Delta f=f_{i n j}-f_{x}$.
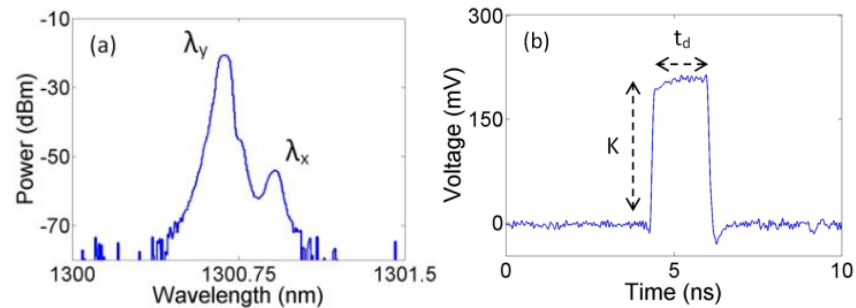

Fig. 2. (a) Optical spectra of the $1300 \mathrm{~nm}-\mathrm{VCSEL}$ driven with $2.0 \mathrm{~mA}$ bias at $293 \mathrm{~K}$. Each polarization mode is labelled accordingly -parallel $\left(\lambda_{y}\right)$ and orthogonal $\left(\lambda_{x}\right)$ modes. (b) Example of an electrically-encoded stimulus with programmable temporal duration $\left(\mathrm{t}_{\mathrm{d}}\right)$ and intensity $(\mathrm{K})$.

An optical isolator (ISO) was included in the setup to avoid undesired reflections to the ML. The power and polarization of the ML's light was controlled using a variable optical attenuator (VOA) and a polarization controller (PC) respectively. A 50/50 fibre coupler was used to divide the optical path into two branches. The first one is connected to a power meter (PM) to monitor the injection strength whilst the second branch is injected into the VCSEL using an optical circulator (CIRC). When polarization matched the external CW injection locked the VCSEL's orthogonal mode to the ML subsequently switching the dominant lasing mode of the VCSEL from parallel to orthogonal polarization. This polarization switching effect, achieved under orthogonallypolarized optical injection, simplified the process of inspecting the injection locking regime and determining the frequency detuning between the VCSEL's injected mode and the external signal. For this reason and the successful achievement of spiking dynamical regimes under the sole injection of optical signals using similar devices [42-43], orthogonal injection with negative frequency detuning was selected for the investigation. Investigation into the injection of parallel polarized light in the vicinity of the main lasing parallel polarization mode of the VCSEL is not discussed in this work. This however may be also considered a feasible avenue of research to obtain similar spiking dynamical responses as those reported in this work. Specially, given the previous demonstration of neuron-like spiking dynamical signals in VCSELs under both parallel and orthogonally polarized 
optical injection [41]. Similarly the effects of electrical injection-induced bistability are not reported in this work. A discussion of bistable regimes induced by orthogonallypolarized injection can be found in a previous work using similar VCSELs at wavelengths of $1550 \mathrm{~nm}$ [50]. In the work presented currently, external injection was typically made with up to $0.5 \mathrm{~mW}$ of injection power and a negative frequency detuning ranging up to $-9 \mathrm{GHz}$. Within this power and detuning range injection locking and the spiking dynamical responses reported in this work could be successfully achieved.

Under these circumstances, external stimuli are encoded in the bias current applied to the device using a bias tee. The bias tee allowed the constant current driving the VCSEL $(6 \mathrm{~mA}$, $\sim 8.57 \mathrm{I}_{\mathrm{th}}$ ) to be combined with the electrically-encoded stimuli. The stimuli were generated in two different ways as depicted graphically in Fig. 1: (1) using directly an electrical signal generator (SG) whose output is connected to the Bias Tee or (2) converting an incoming amplitude-modulated optical signal to the electrical domain using a fast $9.5 \mathrm{GHz}$ amplified photodetector (PHOTO) and adding this resulting electrical signal to the bias current of the device using the bias tee. Here, the amplitude modulated signals were generated using a second tunable laser and a $10 \mathrm{GHz}$ Mach-Zehnder modulator driven by the SG. Additionally, another ISO, VOA and PC were also included to avoid reflections and to control the strength and polarization of the optical signals generating the external stimuli before their conversion to the electrical domain with the PHOTO for their electrical injection into the VCSEL via the bias tee. In both demonstrations the electrical signals imposed on the VCSEL are considered external stimuli as they are representative of stimuli from a neuron influencing another neighbouring synapsed neuron. The second case (2) was specifically investigated to effectively demonstrate the potential implementation of this technique in a future neuroinspired processing network of cascadable, wavelengthindependent, photonic devices such as artificial VCSEL neurons._Hence, for this second case multiple wavelengths were used to demonstrate the wavelength independent nature of the spike activating stimulation.

In both cases analysed in this work, the electrical stimuli encoded in the applied bias current were fully controllable by programming the temporal duration $\left(\mathrm{t}_{\mathrm{d}}\right)$ and intensity $(\mathrm{K})$ of the signal generator (see Fig 2(b)). Using these techniques we were capable of generating electrical stimuli with controlled temporal duration ranging from $t_{d}<1$ ns to $10 \mathrm{~ns}$ and with desired intensity levels. These electrical stimuli triggered short temporal modulations of the device's bias current, causing it to suddenly increase by an amount (current modulation intensity, $I_{p}$ ) determined by perturbation intensity $K$, for the entirety of the perturbation's duration $\left(t_{d}\right)$. This in turn altered the lasing wavelength of the VCSEL resulting in the momentary breakdown of the injection locking condition and the triggering of a spike firing dynamical response at the VCSEL's output. The latter was collected at the exit port of the optical circulator and analyzed spectrally using an optical spectrum analyzer (OSA, Anritsu MS9710B) and temporally using a second amplified high bandwidth (>9.5 GHz) photodetector and a fast real-time $13 \mathrm{GHz}$ oscilloscope (SCOPE, Keysight DSO81304B). For all the results shown throughout this work voltage time series, taken directly from the oscilloscope, were used to clearly demonstrate the dynamics being produced by VCSEL. The established technique of temporal mapping was also used to demonstrate simply the consistency of the reproducible non-linear neuronal dynamics.

\section{EXPERIMENTAL RESULTS}

Figs. 3(a)-(d) show the results obtained at the VCSEL's output for the case of direct injection of electrical stimuli with increasing temporal duration $t_{d}$, (from $1.07 \mathrm{~ns}$, to $1.34 \mathrm{~ns}, 1.90$ ns and $2.35 \mathrm{~ns}$ ) into the device's applied bias current. The electrical stimuli directly injected into the device from the signal generator via a bias tee had a constant intensity $(\mathrm{K}=$ $186 \mathrm{mV}$ ) corresponding to a constant current modulation intensity of $\mathrm{I}_{\mathrm{p}}=3.72 \mathrm{~mA}$ with a set repetition rate of $15 \mathrm{MHz}$. Additionally, the VCSEL was also subject to CW OI with fixed injection strength of $0.252 \mathrm{~mW}$ and a set frequency detuning equal to $-5.65 \mathrm{GHz}$ respectively.

As expected, the VCSEL, under these conditions, is initially injection-locked to the ML's signal yielding a stable temporal output, until the arrival of an electrically-injected stimulus forces the system out of equilibrium, producing as a result a spiking dynamical response. The time window of the incoming stimuli, indicated by black solid lines in Figs. 3(a)(d), can be also clearly identified in the time series of Fig. 3 through the jump in intensity level, associated with the increased power output at higher bias current. During the stimuli time window, consistent $\sim 100$ ps long spikes with subnanosecond inter-spike-intervals are generated. As shown in Figs. 3(a)-(d) the increasing perturbation $t_{d}$ (from $1.07 \mathrm{~ns}$ to $2.35 \mathrm{~ns}$ ) has an effect on the number of generated spikes with the number of spiking events increasing from 1 to 4 as the stimuli duration is extended. Fig. 4 plots in turn merged temporal maps of the VCSEL's responses shown in Figs. 3(a)(d). These maps plot the intensity profile of the system's response to 40 consecutive stimuli (at $15 \mathrm{MHz}$ modulation rate or $66.66 \mathrm{~ns}$ temporal gap between consecutive incoming stimuli) for the four cases of $t_{d}$ investigated. These maps depict low intensity outputs in blue and high intensity spiking outputs in red. Fig. 4 demonstrates the consistency of the achieved spiking dynamics in all cases, producing analogous responses for all incoming perturbations. The temporal plots in Fig. 4 again reveal that the number of spiking events consistently increases with stimuli temporal duration. Increasing the stimulation duration forces the system out of equilibrium and into the spiking regime for a longer period of time making the system trigger the firing of more spiking responses. This behaviour is comparable to the so-called tonic spiking response of biological neurons. For that neuronal regime, spiking events are continuously fired for the whole duration of the stimulation with a total spike count depending on both the $t_{d}$ of the stimulation as well as the specific interspike-interval (or spike rate). The inter-spike-interval is 
defined here as the intrinsic time between individual consecutive spikes in a train of continuously fired spikes. Hence using this method of electrically-activated photonic spiking regimes in VCSELs it is possible to produce controllable trains of spiking dynamics characterized by both sub-nanosecond durations and inter-spike-intervals.

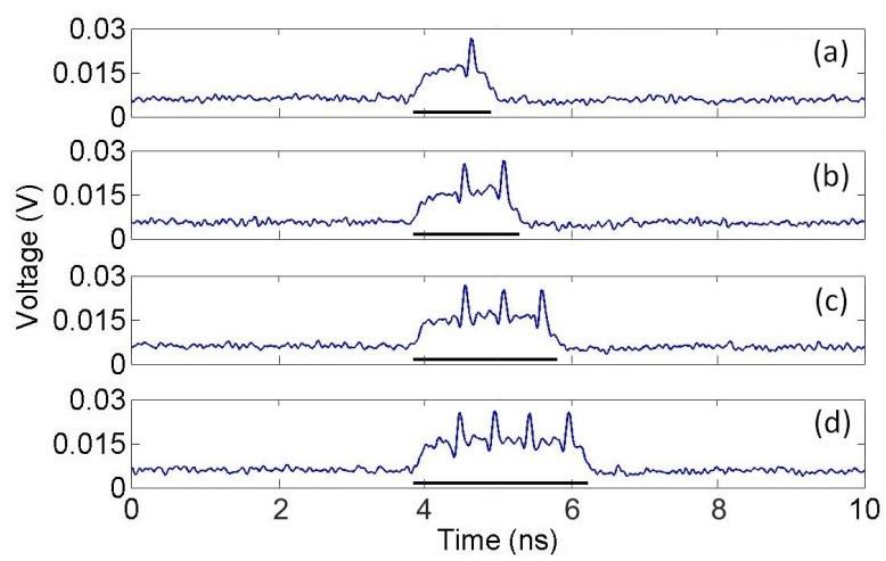

Fig. 3. Time series at the VCSEL's output when an electrically-encoded stimulus with increasing temporal duration $t_{d}$ is injected into its applied bias current: $t_{d}$ is set equal to: (a) $1.07 \mathrm{~ns}$, (b) $1.34 \mathrm{~ns}$, (c) $1.90 \mathrm{~ns}$ and (d) $2.35 \mathrm{~ns}$.

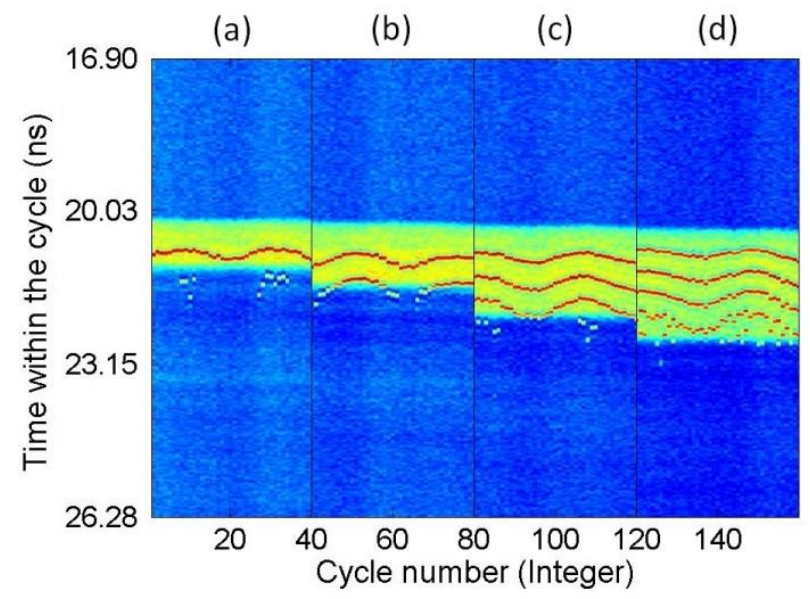

Fig. 4. Merged temporal maps of the VCSEL's output across 40 consecutive electricallyinjected stimuli with increasing t $\mathrm{t}_{\mathrm{d}}$ (a) $1.07 \mathrm{~ns}$, (b) $1.34 \mathrm{~ns}$, (c) $1.90 \mathrm{~ns}$ and (d) $2.35 \mathrm{~ns}$.

The effect of the stimuli intensity $\mathrm{K}$ (translated into the applied modulation intensity $\mathrm{I}_{\mathrm{p}}$, applied to the VCSEL) on the spiking dynamics was also studied to investigate response consistency and spike latency. Fig. 5(a)-(d) shows the time series measured at the VCSEL's output for increasing stimuli strength (modulation intensities $\mathrm{I}_{\mathrm{p}}$ from $1.18 \mathrm{~mA}$ to $4.13 \mathrm{~mA}$ ). The electrical stimuli, $t_{d}=0.65 \mathrm{~ns}$ was selected such that a single spiking response from the system was produced. The stimulus time window is indicated by a black solid line in Fig. 5. The VCSEL was subject to constant orthogonally-polarized optical injection with $0.210 \mathrm{~mW}$ of input strength at a detuning of $\Delta f=-5.65 \mathrm{GHz}$ with respect to the device's orthogonal mode. Fig. 5(a) shows initially the response from the VCSEL when an electrical stimulus of $\mathrm{I}_{\mathrm{p}}=1.18 \mathrm{~mA}$ is injected into the device. In this first case, the device responds by simply increasing its output power as a result of the increased bias current during the stimulus window, but no spiking dynamics are produced by the system. This result is in contrast to the response when an electrical stimulation produces a higher modulation intensity in excess of $\mathrm{I}_{\mathrm{p}}=1.82$ $\mathrm{mA}$ as shown in Figs. 5(b)-(e). Now, the VCSEL reacts by firing a spike event following the incoming stimuli. This is further confirmed in the merged temporal maps plotted in Fig. 6 , showing the response of the VCSEL to 40 consecutive stimuli for each of the five cases depicted in Figs. 5(a)-(e). The maps in Fig. 6 confirm that at low stimulus strength (low modulation intensity) no spiking events are consistently produced, but at high modulation intensities (e), spikes are produced with high consistency. Hence, like in biological neurons, the spiking dynamics in this optoelectronic system require super-threshold stimulus intensity before spike firing is achieved.

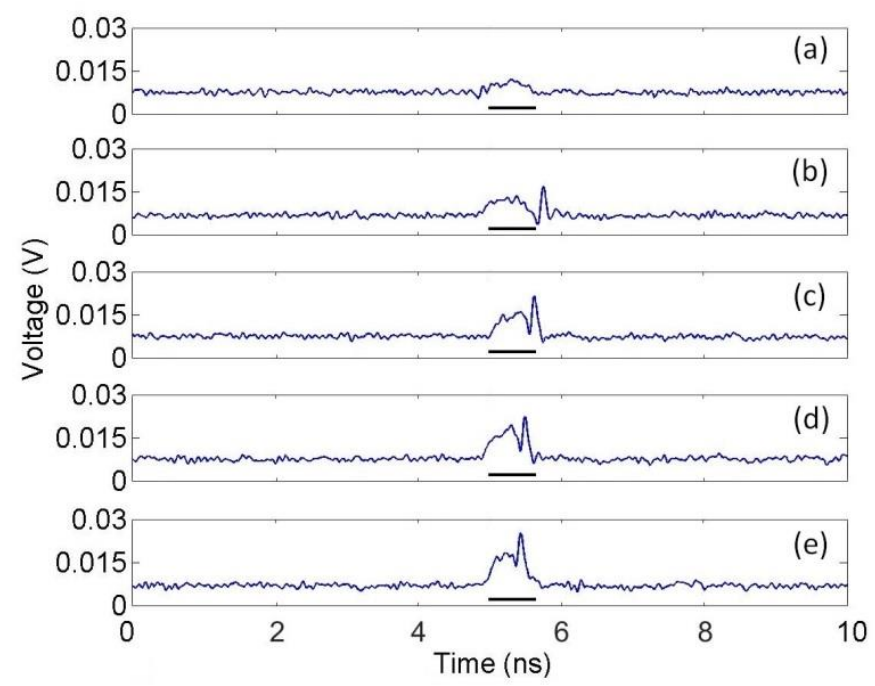

Fig. 5. Time series measured at the VCSEL's output when an electrically-encoded stimulus with increasing strength is injected into its applied bias current. The modulation intensity, $\mathrm{I}_{\mathrm{p}}$ grows from, (a) $1.18 \mathrm{~mA}$ to (b) $1.82 \mathrm{~mA}$, (c) $2.46 \mathrm{~mA}$, (d) $3.33 \mathrm{~mA}$ and (e) $4.13 \mathrm{~mA}$.

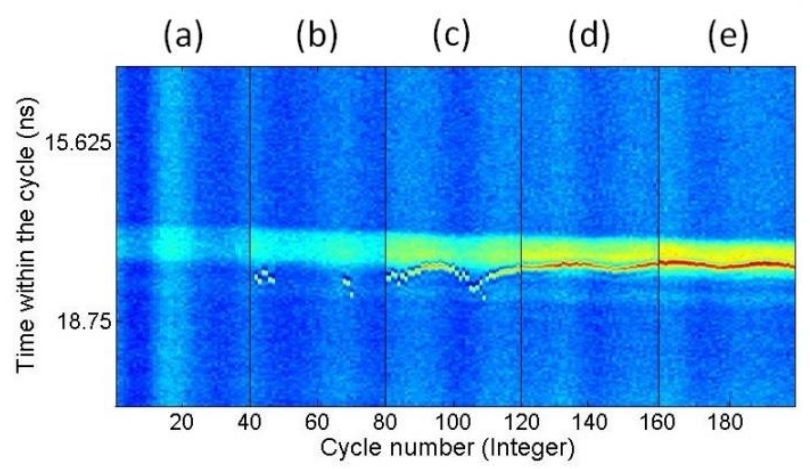

Fig. 6. Merged temporal maps of the VCSEL's output across 40 consecutive electricallyinjected stimuli with increasing strength. The modulation intensity, $\mathrm{I}_{\mathrm{p}}$ grows from, (a) $1.18 \mathrm{~mA}$ to (b) $1.82 \mathrm{~mA}$, (c) $2.46 \mathrm{~mA}$, (d) $3.33 \mathrm{~mA}$ and (e) $4.13 \mathrm{~mA}$.

Varying the stimulus strength also has the effect of altering the spike latency. As seen in Figs. 5(b)-(e) and in the maps in Fig. 6 the spike is fired earlier with less delay in time when increasing the stimulus strength. The behaviour of storing information in the temporal latency (delay) of spike trains has been demonstrated in biological neurons. Neurons in the visual cortex store contrast information in spike latency with 
higher contrast ratios resulting in shorter latency times [51] and auditory signals in neurons were found to contain directionality information in latency times [52]. Similarly this VCSEL neuronal-model encodes information about the strength of the stimulation in the latency of the spikes with a higher strength reducing the latency time experienced by the initial spiking event. With increasing stimulation strength the inter-spike-interval of the responses are also decreased however in this result, because of the short $t_{d}$ selected, this effect is not observed. The short time required to build up carrier dynamics in the device's active region is believed to be responsible for the spike latency and inter-spiking-interval. The stronger a perturbation the faster the evolution of the carrier dynamics and the shorter the spike latency and interspike-interval of the achieved dynamics in the system. This method of electrically-activating fast spiking dynamics in VCSELs therefore also allows VCSELs to produce neuronal threshold dependent, and stimulation-information storing spiking dynamics.

Additionally, we have also tested the wavelengthindependent nature of this scheme (using the red dashed boxed part of the experimental setup in Fig. 1). The main goal was to demonstrate the ultrafast activation of spiking regimes in VCSEL neuronal models with wavelength independent protocols towards future VCSEL-based interconnected neuromorphic processing networks. To carry out this investigation, wavelength-variable and amplitude modulated optical signals were converted to the electrical domain before being electrically encoded (as external stimuli) in the VCSEL's bias current. For our tests on the wavelengthindependent activation of sub-nanosecond spiking dynamics two disparate wavelengths located at different positions in the so-called O-Band were used, namely $1307 \mathrm{~nm}$ and $1290 \mathrm{~nm}$ respectively. In the case of $1307 \mathrm{~nm}$ light, stimuli with increasing temporal durations and constant strength were generated optically and converted to the electrical domain before their injection into the device. Conversely in the case of $1290 \mathrm{~nm}$ light, stimuli of increasing injection strength and constant temporal duration are converted to electrical signals and injected into the device. Fig. 7 shows the response of the VCSEL for the case of $1307 \mathrm{~nm}$ electrically-converted optical stimulation. The device was subject to constant optical injection with a power of $0.155 \mathrm{~mW}$ and an initial detuning of $\Delta f=-7.42 \mathrm{GHz}$ (with respect to the VCSEL's orthogonal mode). For the results in Fig. 7(a)-(d) the temporal window of the incoming stimuli was increased from $1.24 \mathrm{~ns}$, to $1.69 \mathrm{~ns}$, $1.87 \mathrm{~ns}$ and $2.41 \mathrm{~ns}$. The time window of the arriving stimuli is indicated in Fig. 7 with a black solid line. As expected, the electrically-converted optical stimulations produced a similar behaviour to that of the electrical signals shown previously in Fig. 4. Continuous and consistent spiking dynamics were achieved for the duration of the modulation again with the number of spike events generated increasing from 1 in Fig. 7(a) to 4 in Fig. 7(d). This shows that the same controllable neural dynamics can successfully be reproduced using opticalto-electrical converted stimulation; hence opening the door to the interconnection of multiple VCSEL-neuronal models for future network functionalities.

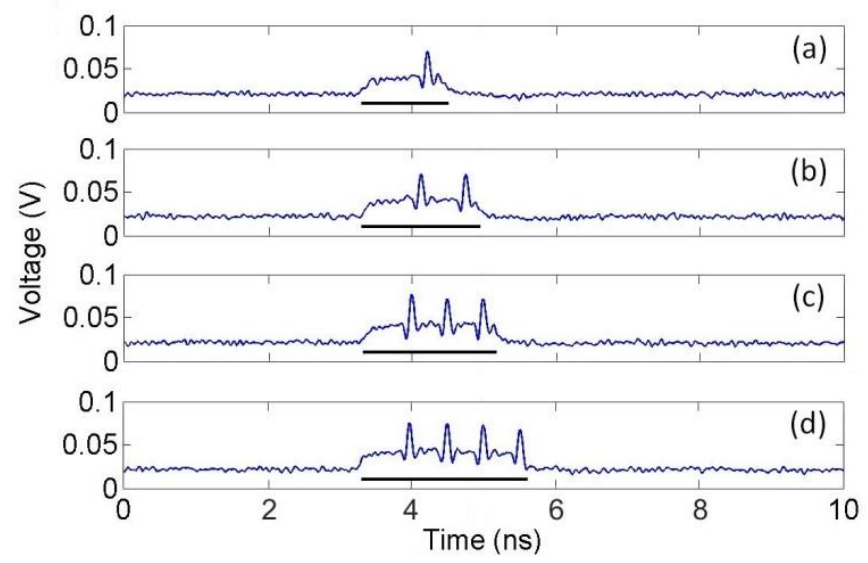

Fig. 7. VCSEL output achieved when $1307 \mathrm{~nm}$ optical-to-electrical converted stimuli of increasing $\mathrm{t}_{\mathrm{d}}$ (a) $1.24 \mathrm{~ns}$, (b) 1.69 , (c) $1.87 \mathrm{~ns}$ and (d) $2.41 \mathrm{~ns}$, are used to electrically trigger the spiking dynamics.

Fig. 8 shows in turn the response obtained from the VCSEL when light of $1290 \mathrm{~nm}$ is encoded with controllable stimuli, converted to the electrical domain and used to electrically activate spiking dynamics in the device. For the results in Figs. 8(a)-(c) the incoming stimuli were set with different injection strength values (the modulation intensities were raised from $2.52 \mathrm{~mA}$ to $3.5 \mathrm{~mA}$ as indicated in the figure caption) whereas their temporal duration, indicated with a black solid line, was kept equal to $2 \mathrm{~ns}$ in all cases. The device was again subject to constant optical injection at $\Delta f=-4.6$ $\mathrm{GHz}$ (with respect to the orthogonal mode of the device) with $0.15 \mathrm{~mW}$ of injection power. Again, and as expected, using now $1290 \mathrm{~nm}$ electrically-converted optical stimulation, spiking dynamics were successfully triggered at the output of the VCSEL. Further, Fig. 8 shows that the number of spikes obtained at the device's output grows from 1 to 3 as the injection strength of the incoming stimulus is grown. This feature is a demonstration of the diminishing inter-spikeinterval as a result of increasing stimulation strength. The increasing stimulation strength shrinks the interval-spikeinterval and in parallel reduces the latency of the spiking. The combined effect of these neuronal behaviours is an increase in the number of spiking responses from the VCSEL. Both of these neuronal behaviours are used by biological neurons to encode information in trains of spiking dynamics. Hence, the VCSEL-based technique reported here is capable of encoding stimulation strength information in spike latency and spike rate similar to biological neurons in the brain. Further, in both cases investigated in Figs. 7 and 8, consistent neural tonic spiking was achieved with spiking dynamics obtained for as long as the stimuli are present; hence demonstrating wavelength-independent activation of neuronal spiking dynamics at ultrafast rates in VCSEL-based photonic neuronal models. This controllable method of activating neural spiking dynamics using wavelength-independent optical signals could help integrate spike producing VCSELs into larger optical 
networks and allow for easier protocols for the future cascadability of spiking photonic neuromorphic systems.

Furthermore, the results in Figs. 3-8 reveal in all cases the generation of sub-ns long spikes (approx. 100 ps long) with inter-spiking intervals also below $1 \mathrm{~ns}$, even if the stimuli are encoded in the electrical current driving the VCSEL. These speed rates are analogous to those obtained in VCSEL neuronal models using wavelength controlled optical stimuli to trigger the spiking responses [26-29][41-43]. Also, the achieved speed rates in this work are faster than those obtained with alternative approaches for spiking optical neuronal models using VCSELs [25][49], or other types of SLs (see [30] and references therein) also relying on electronically injected stimuli. This is due to the fact that in the present approach the electronically-encoded stimuli trigger a transition between an injection-locked and an unlocked regime of operation in the VCSEL (subject to external CW optical injection), which results in the firing of a spiking response. The associated carrier dynamics of this injection locked-unlocked transition, that ultimately determine the achievable inter-spike intervals or recovery time, are faster than the carrier dynamics associated to the transitions between lasing and non-lasing regimes used in other SL-based approaches for optical neuronal models. Using the electricallycontrolled VCSEL neuronal model in this work we should be able to replicate the spike communication networks previously created using the all-optical stimulation approach $[43,44]$. This works new approach, with the additional benefit of being wavelength-independent, could hopefully be expanded into diverse networks of artificial VCSELs neurons capable of processing all-optical spikes similar to the brain.

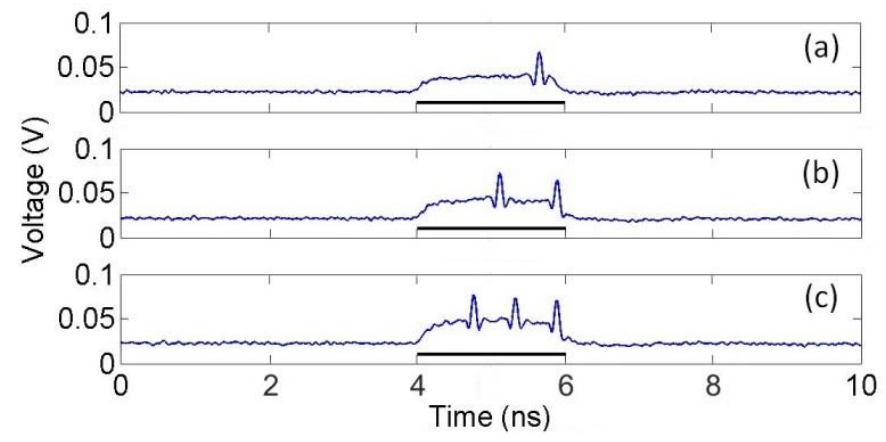

Fig. 8. VCSEL output achieved when $1290 \mathrm{~nm}$ optical-to-electrical converted stimuli of increasing strength are used to electrically trigger the spiking dynamics. The modulation intensity, $\mathrm{I}_{\mathrm{p}}$, is set equal to (a) $2.52 \mathrm{~mA}$, (b) $3.02 \mathrm{~mA}$ and (c) $3.5 \mathrm{~mA}$.

\section{CONCLUSIONS}

In summary, in this work we demonstrate experimentally the controllable generation of spiking regimes at sub-ns speed rates in a VCSEL-based artificial optical neuronal model. These generated spiking signals are over 7 orders of magnitude faster than those produced by biological neurons (millisecond timescales). Moreover, we show that using this technique the VCSEL-based neuronal model studied here can controllably display multiple neuronal behaviours such as tonic spiking, thresholding and the encoding of the stimulus information in the spike latency or firing rate. These spiking dynamics are controllably generated in the device by encoding external perturbations (stimuli) in the VCSEL's applied bias current while subject to constant external optical injection. Under this technique, the constant injection sets the VCSEL in an injection locked state, which is disturbed by the arrival of the electrically-encoded stimuli resulting in the achievement of a spike firing output. Using this technique ultrafast optical spike firing regimes with both sub-nanosecond spike duration and inter-spike time intervals can be generated, given the faster dynamics of the injection locking-unlocking transitions responsible for this behaviour. We show that both directly generated electrical stimuli and electrically-converted, wavelength-independent, optical stimuli can be used to controllably activate the spiking responses in the VCSEL. This technique provides an alternative way of using electrical stimulation to activate spiking dynamics in VCSEL-based photonic neuronal models at very fast speeds (sub-nanosecond rates) compared to previous realizations solely based on optical stimulation, while also offering great prospects for future cascadibility of systems operating at diverse wavelengths. This highlights the versatility of VCSELs as artificial neuronal models for use in neuromorphic platforms and demonstrates the potential these devices have towards creating novel brain-inspired processing architectures for future paradigms in brain-inspired computing and artificial intelligence.

\section{REFERENCES}

[1] B. Benjamin, P. Gao, E. McQuinn, et al., "Neurogrid: A Mixed-AnalogDigital Multichip System for Large-Scale Neural Simulations," Proc. IEEE, vol. 102, pp. 699-716, May (2014).

[2] J. Schemmel, D. Brüderle, A. Grübl, et al., "A Wafer-Scale Neuromorphic Hardware System for Large-Scale Neural Modeling," in Proc. IEEE International Symposium on Circuits and Systems, pp. 1947-1950, May (2010).

[3] P. A. Merolla, J. V. Arthur, R. Alvarez-Icaza, et al., "A Million SpikingNeuron Integrated Circuit with a Scalable Communication Network and Interface," Science, vol. 345, pp. 668-672, Aug (2014).

[4] S. Furber, F. Galluppi, S. Temple, and L. Plana, "The SPiNNaker Project," Proc. IEEE, vol. 102, pp. 652-665, Feb (2014).

[5] E. C. Mos, J. L. Hoppenbrouwers, M. T. Hill, et al., "Optical Neuron by Use of a Laser Diode with Injection Seeding and External Optical Feedback," IEEE Trans. Neural Netw., vol. 11, pp. 988-996, Jul (2000).

[6] A. M. Yacomotti, P. Monnier, F. Raineri, et al., "Fast Thermo-Optical Excitability in a Two-Dimensional Photonic Crystal," Phys. Rev. Lett., vol. 97, 143904, Oct (2006).

[7] M. Brunstein, A. M. Yacomotti, I. Sagnes, et al., "Excitability and Selfpulsating in a Photonic Crystal Nanocavity," Phys. Rev. A, vol. 85, 031803, Mar (2012).

[8] B. Romeira, J. Javaloyes, C. N. Ironside, et al., "Excitability and optical pulse generation in semiconductor lasers driven by resonant tunneling diode photodetectors," Opt. Exp., vol. 21(18), pp. 20931-20940, (2013).

[9] B. Romeira, J. M. L. Figueiredo and J. Javaloyes, "Delay dynamics of neuromorphic optoelectronic nanoscale resonators: perpectives and applications," Chaos, vol. 27, 114323, Nov (2017).

[10] B. J. Shastri, M. A. Nahmias, A. N. Tait, et al., "SIMPEL: circuit model for photonic spike processing laser neurons," Opt. Exp., vol. 23, pp. 8029-8044, (2015).

[11] B. J. Shastri, M. A. Nahmias, A. N. Tait, et al., "Spike processing with a graphene excitable laser," Sci. Rep., vol. 6, 19126, Jan (2016).

[12] K. Kravtsov, M. P. Fok, D. Rosenbluth, and P. R. Prucnal, "Ultrafast alloptical implementation of a leaky integrate-and-fire neuron," Opt. Exp., vol. 19, pp. 2133-2147, (2011).

[13] R. Toole, A. N. Tait, T. Ferreira de Lima, et al., "Photonic Implementation of Spike-Timing-Dependant Plasticity and Learning 
Algorithms of Biological Neural Systems," J. Lightwave Technol., vol. 34, pp. 470-476, (2016).

[14] A. Tait, T. Ferreira de Lima, E. Zhou, et al., "Neuromorphic photonic networks using silicon photonics weight banks," Sci. Rep., vol. 7, 7430, (2017).

[15] M. P. Fok, Y. Tian, D. Rosenbluth, and P. R. Prucnal, "Asynchronous spiking photonic neuron for lightwave neuromorphic signal processing," Opt. Lett., vol. 37, pp. 3309-3311, (2012).

[16] K. Alexander, T. Van Vaerenbergh, M. Fiers, et al., "Excitability in optically injected microdisk lasers with phase controlled excitatory and inhibitory response," Opt. Exp., vol. 21, pp. 26182-26191, (2013).

[17] S. Barbay, R. Kuszelewicz, and A. M. Yacomotti, "Excitability in a semiconductor laser with saturable absorber," Opt. Lett., vol. 36, 44764478, (2011)

[18] F. Selmi, R. Braive, G. Beaudoin, et al., "Relative Refractory Period in an Excitable Semiconductor Laser," Phys. Rev. Lett., vol. 112, 183902, May (2014).

[19] F. Selmi, R. Braive, G. Beaudoin, et al., "Spike latency and response properties of an excitable micropillar laser," Phys. Rev. E, vol. 94, 042219, Oct (2016).

[20] W. Coomans, L. Gelens, S. Beri, et al., "Solitary and coupled semiconductor ring lasers as optical spiking neurons," Phys. Rev. E, vol. 84, 036209, Sept (2011).

[21] L. Gelens, L. Mashal, S. Beri, et al., "Excitability in semiconductor microring lasers: Experimental and theoretical pulse characterization," Phys. Rev. A, vol. 82, 063841, Dec (2010).

[22] C. Mesaritakis, A. Kapsalis, A. Bogris and D. Syvridis, "Artificial Neuron Based on Integrated Semiconductor Quantum Dot Mode-Locked Lasers," Sci. Rep., vol. 6, 39317, Dec (2016).

[23] B. Kelleher, C. Bonatto, G. Huyet, and S. P. Hegarty, "Excitability in optically injected semiconductor lasers: Contrasting quantum-well- and quantum-dot-based device," Phys. Rev. E, vol. 83, 026207, Feb (2011).

[24] B. Kelleher, C. Bonatto, P. Skoda, et al., "Excitation regeneration in delay-coupled oscillators," Phys. Rev. E, vol. 81, 036204, Mar (2010).

[25] M. A. Nahmias, B. J. Shastri, A. N. Tait and P. R. Prucnal, "A Leaky Integrate-and-Fire Laser Neuron for Ultrafast Cognitive Computing," IEEE J. Sel. Top. Quantum Electron., vol. 19, 1800212, Sep (2013)

[26] M. Turconi, B. Garbin, M. Feyereisen, et al., "Control of excitable pulses in an injection-locked semiconductor laser," Phys. Rev. E, vol. 88, 022923, (2013).

[27] B. Garbin, J. Javaloyes, G. Tissoni and S. Barland, "Topological solitons as addressable bits in a driven laser," Nat. Commun., vol. 6, 5915, Jan (2015).

[28] A. Hurtado, K. Schires, I. D. Henning and M. J. Adams, "Investigation of vertical cavity surface emitting laser dynamics for neuromorphic photonic systems," Appl. Phys. Lett., vol. 100, 103703, Feb (2012).

[29] S. Xiang, A. Wen and W. Pan, "Emulation of Spiking Response and Spiking Frequency Property in VCSEL-Based Photonic Neuron," IEEE Photon. J., vol. 8, 1504109, Oct (2016).

[30] P. R. Prucnal, B. J. Shastri and T. Ferreira de Lima, "Recent progress in semiconductor excitable lasers for photonic spike processing," Adv. Opt. Photon., vol. 8, pp. 228-299, (2016).

[31] K. Schires, A. Hurtado, I. D. Henning and M. J. Adams, "Rare disruptive events in polarization-resolved dynamics of optically injected 1550 nm VCSELs," Electron. Lett., vol. 48, pp. 872-874, Jul (2012).

[32] R. Al-Seyab, I. D. Henning, M, J, Adams and A. Hurtado, "Controlled single- and multiple-pulse excitability in VCSEs for novel spiking photonic neurons," 2014 IEEE Int. Semicond. Laser Conf., pp. 165-166, Dec (2014).

[33] K. Al-Naimee, F. Marino, M. Ciszak, et al., "Excitability of periodic and chaotic lasers with optoelectronic feedback," Eur. Phys. J. D., vol. 58, pp. 187-189, Apr (2010).

[34] H. J. Wunsche, O. Brox, M. Radziunas, and F. Henneberger, "Excitability of a Semiconductor Laser by a Two-Mode Homoclinic Bifurcation," Phys. Rev. Lett., vol. 88, 023901, Dec (2002).

[35] J. P. Toomey, C. Nichkawade, D. M. Kane, et al., "Stability of the nonlinear dynamics of an optically injected VCSEL," Opt. Exp., vol. 20, pp. 10256-10270, (2012).

[36] A. Hurtado, A. Quirce, A. Valle, et al., "Nonlinear dynamics induced by parallel and orthogonal optical injection in $1550 \mathrm{~nm}$ Vertical-Cavity Surface-Emitting Lasers (VCSELs)," Opt. Exp., vol. 18, pp. 9423-9428, (2010).

[37] M. J. Adams, A. Hurtado, D. Labukhin and I. D. Henning, "Non-linear semiconductor lasers and amplifiers for all-optical information processing," Chaos, vol. 20, 037102, Aug (2010).
[38] F. Koyama, "Recent Advances of VCSEL Photonics," J. Lightwave Technol., vol. 24, pp. 4502-4513, Dec (2006).

[39] A. Hurtado, I. D. Henning and M. J. Adams, "Optical neuron using polarization switching in a 1550nm-VCSEL," Opt. Exp., vol. 18, pp. 25170-25176, (2010).

[40] P. Pérez, A. Valle, L. Pesquera and A. Quirce, "All-Optical Inverter Based on Polarization Switching in VCSELs Subject to Single and Dual Optical Injection," IEEE J. Sel. Top. Quantum Electron., vol. 19, 1700408, Dec (2012).

[41] A. Hurtado and J. Javaloyes, "Controllable spiking patterns in longwavelength vertical cavity surface emitting lasers for neuromorphic photonics systems," Appl. Phys. Lett., vol. 107, 241103, Nov (2015).

[42] J. Robertson, T. Deng, J. Javaloyes and A. Hurtado, "Controlled inhibition of spiking dynamics in VCSELs for neuromorphic photonics: theory and experiments," Opt. Lett., vol. 42, 1560-1563, (2017).

[43] T. Deng, J. Robertson and A. Hurtado, "Controlled propagation of spiking dynamics in vertical-cavity surface-emitting lasers: towards neuromorphic photonic networks," J. Sel. Top. Quantum. Electron., vol. 23, 1800408, Mar (2017).

[44] T. Deng, J. Robertson, Z. M. Wu, et al., "Stable propagation of inhibited spiking dynamics in vertical-avity surface-emitting lasers for neuromorphic photonics networks," IEEE Access, vol. 6, pp. 6795167958, Nov (2018).

[45] S. Xiang, Y. Zhang, X. Guo, et al., "Photonics Generation of NeuronLike Dynamics Using VCSELs Subject to Double Polarized Optical Injection," J. Lightwave. Technol., vol. 36, pp. 4227-4234, (2018).

[46] Y. Zhang, S. Xiang, X. Guo, et al., "Polarization-resolved and polarization-multiplexed spike encoding properties in photonic neuron based on VCSEL-SA," Sci. Rep., vol. 8, 16095, Dec (2018).

[47] H. Susanto, K. Schires, M. J. Adams and I. D. Henning, "Spin-flip model of spin-polarized vertical-cavity surface-emitting lasers: Asymptotic analysis, numerics and experiments," Phys. Rev. A, vol. 92, 063838, Dec (2015).

[48] S. Xiang, H. Zhang, X. Guo, et al., "Cascadable neuron-like spiking dynamics in coupled VCSELs subject to orthogonally polarized optical pulse injection," J. Sel. Top. Quantum. Electron., vol. 23, 1700207, Mar (2017).

[49] Y. Zhang, S. Xiang, J. Gong, et al., "Spike encoding and storage properties in mutually coupled vertical-cavity surface-emitting lasers subject to optical pulse injection," Appl. Opt., vol. 57, pp. 1731-1737, (2018).

[50] A. Hurtado, A. Quirce, A. Valle, et al., "Power and wavelength polarization bistability with very wide hysteresis cycles in a $1550 \mathrm{~nm}-$ vcsel subject to orthogonal optical injection," Opt. Exp., vol 17, pp. 23637-23642, (2009).

[51] D. S. Reich, F. Mechler and J. D. Victor, "Temporal coding of contrast in primary visual cortex: when, what and why," J. Neurophysio., vol. 85, pp. 1039-1050, (2001).

[52] S. Furukawa and J. C. Middlebrooks, "Cortical representation of auditory space: information-bearing features of spike patterns," J. Neurophysio., vol. 87, pp. 1749-1762, (2002).

Joshua Robertson was born in Glasgow, U.K. in 1994. He received his MPhys degree in Physics with specialization in Photonics from the University of Strathclyde, Glasgow in 2017. He is currently working towards his Ph.D. degree at the Institute of Photonics, University of Strathclyde, Glasgow, focusing on neuromorphic photonic systems with lasers.

Ewan Wade was born in Glasgow, U.K. in 1996. He is currently working towards his MPhys degree in Physics with specialization in Photonics from the University of Strathclyde, Glasgow.

Antonio Hurtado received the Ph.D. degree from the Universidad Politécnica de Madrid (UPM), Madrid, Spain in December 2006. He has more than 10 years' international research experience in photonics in the UK (Universities of Essex and Strathclyde), USA (University of New Mexico), and Spain (UPM). He received two Marie Curie Fellowships by the European Commission: Projects ISLAS (2009-2011) \& NINFA (2011-2014). In 2014 he was awarded Chancellor's Fellowship by the University of Strathclyde following which he was appointed as a Lecturer (Assist. Prof.) in the Strathclyde's Institute of Photonics (Physics Dept.). He was promoted to Senior Lecturer (Assoc. Prof.) in 2018. His current research interests are but not limited to neuromorphic photonics, laser nonlinear dynamics, nanolaser systems and hybrid nanofabrication. 